Bundesgesundheitsbl 2020 63:176-184 https://doi.org/10.1007/s00103-019-03087-6 (c) Der/die Autor(en) 2020

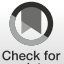
Online publiziert: 16 . Januar 2020

Uwe H. Bittlingmayer ${ }^{1}$ Kevin Dadaczynski ${ }^{2,3}$. Diana Sahrai ${ }^{4}$ Stephan van den Broucke $^{5} \cdot$ Orkan Okan ${ }^{6}$

'Institut für Soziologie, Pädagogische Hochschule Freiburg, Freiburg, Deutschland

${ }^{2}$ Fachbereich Pflege und Gesundheit, Hochschule Fulda, Fulda, Deutschland

${ }^{3}$ Leuphana Universität Lüneburg, Zentrum für Angewandte Gesundheitswissenschaften, Lüneburg, Deutschland

${ }^{4}$ Institut Spezielle Pädagogik und Psychologie, Pädagogische Hochschule FHNW, Muttenz, Schweiz

${ }^{5}$ Psychological Sciences Research Institute, Université catholique de Louvain, Louvain-la-Neuve, Belgien

${ }^{6}$ Fakultät für Erziehungswissenschaft, Universität Bielefeld, Bielefeld, Deutschland

\section{Digitale Gesundheitskompetenz - Konzeptionelle Verortung, Erfassung und Förderung mit Fokus auf Kinder und Jugendliche}

\section{Einleitung}

Digital Health Literacylässt sich zunächst als einer von mehreren Bestandteilen der als umfassender gedachten Health Literacy, oder in der deutschen Übersetzung Gesundheitskompetenz, bestimmen. Im Laufe der letzten 15 Jahre hat sich dabei eine größere Anzahl von Begriffsvorschlägen ausgebreitet: So finden sich neben der Digital Health Literacy [1] die Begriffe E-Health Literacy [2], Media Health Literacy [3], Electronic Health Literacy [4] oder noch spezifischer Internet Health Literacy [5] und Mobile Health Literacy [6]. Im Rahmen des vorliegenden Beitrags werden die feinen Verästelungen dieser Begrifflichkeiten nicht weiterverfolgt, sondern der Begriff „digitale Gesundheitskompetenz" genutzt und die anderen Begriffsvorschläge darunter subsumiert.

In einer ersten Annäherung liegen im Gegenstandsbereich von digitaler Gesundheitskompetenz beispielsweise Motive wie die kompetente und souveräne Navigation durch das Internet im Zusammenhang mit der Beantwortung gesundheitsrelevanter Fragen, die Flankierung eines gesundheitsorientierten Lebensstils bzw. der kompetente Um- gang mit chronischen Erkrankungen durch die Verwendung von gesundheitsbezogenen Apps oder die Erzeugung von Aufmerksamkeit für gesundheitsbezogene Themenstellung in sozialen Medien, etwa durch Partizipation an einer Facebook-Gruppe für die qualitative Verbesserung der industriellen Nahrungsmittelproduktion. Aber es wird schnell deutlich, dass der Gegenstandsbereich von digitaler Gesundheitskompetenz noch erheblich umfassender ist und bereits vor rund zwei Jahrzehnten vor allem mit der Einführung einer elektronischen Patientenakte verbunden wurde. Der Großteil der jüngeren Arbeiten zu digitaler Gesundheitskompetenz bezieht sich aber auf die erheblich angewachsenen individuellen Optionsräume, die mit der Digitalisierung und der damit zusammenhängenden exorbitanten Ausdehnung von verfügbaren werden.

Die versorgungsbezogene Seite digitaler Gesundheitskompetenzen bleibt in diesem Beitrag größtenteils ausgeklammert, weil es sich hier um einen eigenständigen Diskurs handelt. Wir wollen uns in diesem Beitrag stattdessen auf die lebensweltlichen Dimensionen von diGesundheitsinformationen verursacht gitaler Gesundheitskompetenz beziehen und damit der sukzessiven Ausdehnung des gesamten Health-Literacy-Konzepts, wie er sich in neueren und umfassenderen Health-Literacy-Definitionen widerspiegelt [7], folgen. Zudem soll ein besonderer Fokus auf Kindern und Jugendlichen liegen, weil diese Gruppe im Kontext von digitalen Gesundheitskompetenzen sehr widersprüchlich konzeptionalisiert wird: Erstens im Rahmen einer nunmehr heranwachsenden Generation von Digital Natives werden Kinder und Jugendliche als besonders kompetente und souveräne Nutzende der digitalen Welt imaginiert; zweitens werden Kinder und Jugendliche im Rahmen der nunmehr auch von der Weltgesundheitsorganisation (WHO) als psychische Erkrankung anerkannten Internetsucht als besondere Risikogruppe angesehen und drittens zeigen empirischeStudien, dass sich vor allem Jugendliche im Selbstreport als gesundheitskompetenter einschätzen als Erwachsene.

Dieser Beitrag zielt darauf, einen Überblick zu verschiedenen Facetten der digitalen Gesundheitskompetenz im Kindes- und Jugendalter zu vermitteln. Hierfür werden zunächst bestehende Gemeinsamkeiten zwischen Gesundheitsund Medienkompetenzen diskutiert und 
der gesundheitsbezogene Informationszugang über Medien dargestellt, um den Gegenstandbereich der digitalen Gesundheitskompetenz an den medienpädagogischen Diskurs anzuschließen. Darauf folgen die Präsentation vorliegender Konzepte zur digitalen Gesundheitskompetenz sowie eine ungleichheitsorientierte Betrachtung digitaler Aspekte bezüglich der Gesundheit. Im Abschnitt zur Messung der digitalen Gesundheitskompetenz werden sowohl aktuell vorliegende Instrumente vorgestellt als auch die theoretischen Hintergründe der Operationalisierung besprochen. Dieser Beitrag schließt mit einer Schlussbetrachtung und einem Blick auf Interventionen zur Stärkung der digitalen Kompetenzen bei Kindern und Jugendlichen in der Schule und vor dem Hintergrund des digitalen Wandels.

\section{Die Verschränkung von Gesund- heits- und Medienkompetenzen}

Das Thema der digitalen Gesundheitskompetenz ist aus der Health-LiteracyForschung und -Debatte nicht wegzudenken und nimmt einen kontinuierlich prominenteren Stellenwert ein [3, 8-15]. Gerade in Hinblick auf die Gesundheitskompetenz von Kindern und Jugendlichen spielt Digitalisierung eine besonders zentrale Rolle, weil die heranwachsende Generation gewissermaßen mit Geburt in die technischen Möglichkeiten und Errungenschaften des Internets und seine digitalen Weiterentwicklungen hineinsozialisiert wird $[11,13,16-18]$. So sind digitale Medien bereits bei Kindern und Jugendlichen alltägliche Begleiter; kindliche und jugendliche (und folglich auch familiale) Lebenswelten sind vom Internet und den notwendig angeschlossenen, insbesondere mobilen Nutzungsgeräten mehr oder weniger vollständig durchdrungen $[8,9]$. Die Ergebnisse der vom Medienpädagogischen Forschungsverbund Südwest durchgeführten Studien JIM (Jugend, Information, (Multi-)Media) und KIM (Kinder, Internet, Medien) weisen darauf hin, dass etwa die Hälfte aller 6- bis 13-Jährigen und annähernd $100 \%$ aller 12- bis 19-Jährigen ein eigenes Smartphone und etwa $20 \%$ bzw.
$70 \%$ einen eigenen Computer/Laptop besitzen $[19,20]$. Dabei nimmt der Anteil derjenigen, die täglich das Internet nutzen, erwartungsgemäß im Altersgang auf etwa $90 \%$ im Jugendalter zu [19], beträgt jedoch in der Alterskohorte der 6- bis 13-Jährigen bereits $27 \%$ [20].

Im Zusammenhang mit digital gestützten gesundheitsbezogenen Informationsangeboten ist unbestritten, dass historisch gesehen ein neuer Optionsraum geöffnet wurde, was für die individuelle Gesundheit im weitesten Sinne eine Reihe ambivalenter Konsequenzen hat. Für das Verhalten in diesem Optionsraum sind spezielle Kompetenzen notwendig wie etwa die digitalen Gesundheitskompetenzen zur Förderung, Aufrechterhaltung und Wiederherstellung von Gesundheit sowie zur Vermeidung von oder zum Umgang mit Krankheit. Klar ist auch, dass das Internet und die sogenannten sozialen Medien, die mittlerweile ihren Siegeszug vollzogen haben, eine bedeutende Rolle bei der Bereitstellung und auch der Suche von gesundheitsbezogenen Informationen spielen [19-21]. Anders als früher ist also im Zeitalter des Internets auch für Kinder und Jugendliche weniger das prinzipielle Problem der Informationsknappheit und Informationsbereitstellung als vielmehr die präzise Informationssuche sowie die angemessene Informationsbewertung und -selektion immanent, wodurch jedoch zur ohnehin komplexen Thematik individueller Gesundheitskompetenz ein weiteres Komplexitätsniveau hinzutritt.

Denn Studien weisen darauf hin, dass nicht nur das Auffinden von Gesundheitsinformationen relevant ist, sondern eben auch die personale Fähigkeit, deren Verlässlichkeit einschätzen zu können. Exemplarisch angeführt werden kann eine Analyse von 140 YouTube-Videos zum Thema Anorexie durch Medizinerinnen und Mediziner, deren Ergebnisse darauf hinweisen, dass etwa $30 \%$ der gesichteten Beiträge eine anorexiaförderliche Ausrichtung aufwiesen (z.B. Anorexia als Mode und Schönheitsideal [22]). Im Vergleich $\mathrm{zu}$ den informativen Videos erhielten Proanorexiabeiträge eine dreifach höhere Zustimmung (Klickraten und Likes). Vor diesem Hintergrund ist es nicht verwunderlich, dass bereits im Jahr 2014 bei Umfragen in der Europäischen Union im Rahmen des FlashEurobarometers die mangelnde Verlässlichkeit als Hauptgrund für eine geringe Zufriedenheit mit den Ergebnissen der gesundheitsbezogenen Onlinerecherche genannt wurden [23]. In diesem Sinne resümieren auch Paakkari und George [24]:

The ongoing health literacy crisis is not caused by lack of information. ... Neither is the crisis about skills required to gain access to information, as was the case in the late twentieth century when information was limited, not easily available and took different forms. Now, in contrast, the internet's exponential development in a globalized world requires skills to use effectively an abundance of health information and to distinguish between information of varying quality through comparison, classification and assessment of credibility.

Während dieser kritische Hinweis bezüglich der Güte von gesundheitsbezogenen Informationen sicherlich zunächst für die westliche Welt, für Industriestaaten und für Gebiete gilt, die durch das Internet erschlossen sind, müssen für andere Gebiete und Regionen, die vom Internet unerschlossen sind und/oder in denen akute Gesundheitsprobleme andere Maßnahmen erfordern, als durch Informationskompetenzen zu bewältigen wären, differenzierte Lösungsansätze erarbeitet werden. Deutlich scheint für die erstgenannten Länder und Gebiete allemal, dass sich individuelle Gesundheitskompetenzen und (kritische) Medienkompetenzen miteinander verschränken, da die Fähigkeit, (digitale) Medien entsprechend der eigenen Bedürfnisse zu nutzen und mit diesen verantwortungsvoll umzugehen, eine unverzichtbare Voraussetzung darstellt, um deren gesundheitsbezogene Inhalte kritisch-reflexiv erschließen und für die eigene Gesundheit nutzen zu können $[25,26]$. 
Definitionen, Konzeptionalisierung und Verständnis von digitaler Gesundheitskompetenz

Die vorangegangenen Ausführungen belegen zusammengenommen, dass der gesundheitsbezogene Informationszugang über Medien und insbesondere digitale Medien im Kindes- und Jugendalter in den letzten Jahren kontinuierlich an Bedeutung gewonnen haben. Dass Medienkompetenzen eine solch bedeutsame Rolle für die Aufrechterhaltung, Förderung und Wiederherstellung von Gesundheit sowie für gesundheitsbezogenes Verhalten zugesprochen wird, verdeutlicht sich auch an den einschlägigen Definitionsversuchen von digitaler Gesundheitskompetenz (und deren Synonymen). Die am häufigsten verwendeten Definitionen stammen von Eng [27] sowie Norman und Skinner [2]. Eng führt bereits zu Beginn der Health-LiteracyDebatte eine kurze, sehr nahe an gängigen allgemeinen Health-Literacy-Konzepten angelehnte Definition ein, welche digitale Gesundheitskompetenzen bestimmt als die Fähigkeit für die Benutzung von Informations- und Kommunikationstechnologien, insbesondere internetbasierten Technologien, um die Gesundheit und den Zugang zum Versorgungssystem zu verbessern. [27].

Diebis heute diskursbestimmende Arbeit und auch Definition stammt jedoch von Norman und Skinner, die E-Health Literacy nicht nur abstrakt bestimmt, sondern auch konkret operationalisiert haben. Sie beschreiben digitale Gesundheitskompetenz als die Fähigkeit zum Suchen, Finden, Verstehen und Bewerten von Gesundheitsinformationen auf der Grundlage digitaler Quellen und das gewonnene Wissen so anzuwenden, um gesundheitliche Herausforderung $\mathrm{zu}$ adressieren und Probleme zu lösen [2]. Sie konkretisieren digitale Gesundheitskompetenz weiter und kreieren mit dem Lilienmodell einen bis heute häufig verwendeten Standard in der Bestimmung von digitaler Gesundheitskompetenz. In diesem Modell werden sechs basale analytische und kontextspezifische Fähigkeiten zu einem integrierten Kompetenzmodell angeordnet. Diese sind, wie

Bundesgesundheitsbl 2020 -63:176-184 https://doi.org/10.1007/s00103-019-03087-6 (c) Der/die Autor(en) 2020

\section{U. H. Bittlingmayer · K. Dadaczynski · D. Sahrai · S. van den Broucke · 0. Okan}

\section{Digitale Gesundheitskompetenz - Konzeptionelle Verortung, Erfassung und Förderung mit Fokus auf Kinder und Jugendliche}

\section{Zusammenfassung}

Das Konzept der digitalen Gesundheitskompetenz ist das Ergebnis der zunehmenden gesellschaftlichen Durchdringung von digitalen Medien und deren Nutzung im Alltag. Infolge der hohen Zugänglichkeit und Allgegenwärtigkeit von Gesundheitsinformationen steigt die Notwendigkeit nicht nur des Suchens und Findens, sondern insbesondere der Einschätzung ihrer Verlässlichkeit und der darauf bezogenen Selektion und Anwendung für eigene Gesundheitsbelange. Gerade im Kontext der Digitalisierung ist zudem zu berücksichtigen, dass die Nutzenden nicht nur passiv rezipieren, sondern aktiv am Kommunikationsgeschehen durch Interaktion mit bestehenden Inhalten oder durch eigene Informationsbeiträge teilhaben.

Der vorliegende Beitrag gibt eine Übersicht über den aktuellen Forschungsstand zur digitalen Gesundheitskompetenz, wobei der Fokus vor allem auf dem Kindes- und Jugendalter liegt. Neben seiner terminolo- gisch-konzeptionellen Verortung und der notwendigen Verschränkung mit dem Ansatz der Medienkompetenzen wird das Verhältnis von digitaler Gesundheitskompetenz und sozialer sowie gesundheitlicher Ungleichheit betrachtet. Als ungleichheitsgenerierend wird dabei weniger der unterschiedliche Zugang zu digitalen Medien angesehen, sondern vor allem die Unterschiede in den zum Umgang notwendigen Kompetenzen. Nach einer Übersicht über die wenigen bisher verfügbaren Instrumente zur Erfassung der digitalen Gesundheitskompetenz werden am Beispiel des Settings Schule erste Überlegungen zu ihrer individuellen und strukturellen Stärkung angestellt.

\section{Schlüsselwörter}

Digitale Gesundheitskompetenz · Medienkompetenz · Konzept · Operationalisierung . Kinder und Jugendliche

\section{Digital health literacy-conceptual contextualization, measurement, and promotion}

\section{Abstract}

The concept of digital health literacy can be regarded as the result of the increasing social permeation of digital media and their use in everyday life. Due to increasing accessibility and ubiquity, there is an increasing need not only for searching and finding, but especially for assessing the reliability as well as selecting and applying health information for one's own health concerns. In the context of digitization, it needs to be emphasized that users are not just passive recipients, but rather actively participate in the communication process by interacting with existing content or by sharing their own health-related information. With particular focus on children and adolescents, this paper provides an overview of the current state of research on digital health literacy. In addition to its terminological and conceptual foundations and its links with media literacy, the relationship between digital health literacy and social and health inequality is discussed. Inequalities are not only generated by access to digital media but, above all, by the skills required for their use. After an overview of available instruments is provided, initial reflections on how to promote the individual and structural dimension of digital health literacy with focus on school settings is given.

\section{Keywords}

Digital health literacy · Media literacy · Concept - Operationalization · Children and adolescents aus • Abb. 1 ersichtlich, zunächst als analytische Komponenten (Dimensionen) definiert: „Traditional Literacy“ (Literalität im Sinne von Alphabetisierung und Grundbildung; Lese- und Rechtschreibkompetenz), die sich auf das formale Bildungsniveau, Lese-, Rechtschreib- und alltagsmathematische Rechenkompetenzen bezieht, sowie „Information Literacy“ (Informationskompetenz) und „Media Literacy“ (Medienkompetenz), die bereits anwendungsorientierte Kompetenzen bezeichnen, die auf den Umgang mit Informationen und neuen 


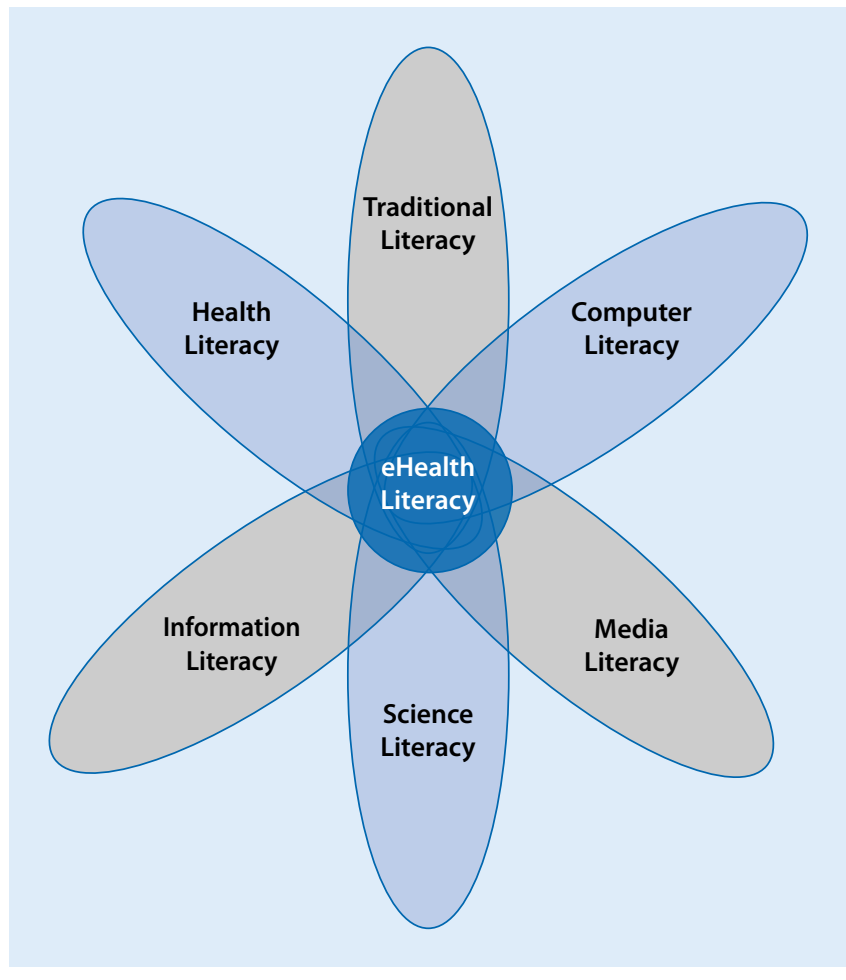

Abb. $1<$ Digitale Gesundheitskompetenz (E-Health Literacy) und zugrunde liegende Fähigkeiten. (Adaptiert nach Lilienmodell von Norman und Skinner, 2006 https://www.jmir. org/2006/2/e9/ [2], @ Cameron D Norman, Harvey A Skinner; publiziert unter der CC-BY 2.0 Lizenz: https:// creativecommons. org/licenses/by/2. $0 /)$

Medien ganz im Sinne der von Baacke [28] definierten Dimension Medienkunde abzielen. Über diese Dimensionen hinaus gehen als kontextspezifische bzw. kontextsensitive Kompetenzen ferner direkte Nutzerkompetenzen ein, die als "Computer Literacy“ (Computerkompetenz) verstanden werden. Stark ist der Einbezug von "Science Literacy“ (Wissenschaftskompetenz), die sich auf ein biopsychosoziales Verständnis von Gesundheit und die Fähigkeit beruft, wissenschaftliche Befunde $\mathrm{zu}$ verstehen, zu bewerten und zu interpretieren. Diese Kompetenz ist äußerst voraussetzungsreich gefasst: „For those who do not have the educational experience of exposure to scientific thought, understanding science-based online health information may present a formidable challenge. Science literacy places health research findings in appropriate context, allowing consumers to understand how science is done, the largely incremental process of discovery, and the limitations - and opportunities - that research can present" [2].

Die letzte Dimension ist Gesundheitskompetenz, die recht orthodox verstanden wird als der Vollzug wohlinformierter individueller Gesundheits- entscheidungen. Das Lilienmodell ist bis heute besonders populär. So führt etwa Bautista [29] aus, dass die Definition von Norman und Skinner die im Kontext von digitaler Gesundheitskompetenz am häufigsten verwendete ist. Allerdings ist das Lilienmodell aber mittlerweile auch substanziell in die Kritik geraten. So wurde zum Beispiel die handlungstheoretische Grundlegung als $\mathrm{zu}$ individuumzentriert bemängelt. Insbesondere Gilstad [30] arbeitet die subjektbezogene Verkürzung heraus. Sie schlägt in diesem Zusammenhang vor, weitere Kompetenzen wie kontextbezogene und kommunikative Kompetenzen einzubeziehen, und erweitert das Lilienmodell um den soziokulturellen sowie situationalen Kontext. Gilstad zufolge beeinflussen insbesondere soziale Faktoren wie gesellschaftliche Normen, Glaubenshorizonte und geteilte Vorstellungen und Werte maßgeblich die Nutzung digitaler Gesundheitsangebote.

Gerade die Betonung der sozialen und kulturellen Dimensionen der Technologienutzung hat die Diskussion seit Norman und Skinner weitergeführt und zu einer umfassenderen Definition digitaler Gesundheitskompetenzen beigetragen, die erheblich deutlicher auf die soziale Einbettung individueller Techniknutzung abhebt. Bautista [29; eigene Übersetzung] liefert die folgende bislang umfassendste Definition digitaler Gesundheitskompetenz: „Elektronische Gesundheitskompetenz (E-Health Literacy) umfasst das Zusammenspiel personaler und sozialer Faktoren bei der Nutzung digitaler Technologien im Suchen, Aneignen, Erfassen, Verstehen, Bewerten, Kommunizieren und Anwenden von Gesundheitsinformationen in allen Kontexten der Gesundheitsversorgung mit dem Ziel, die Lebensqualität über die gesamte Lebensdauer hinweg zu erhalten oder zu verbessern."

Die Betonung der sozialen Einbettung von Techniknutzung ist gerade für eine gesundheitsbezogene Verwendung etwa von Smartphones im Kindes- und Jugendalter aus unserer Sicht besonders bedeutsam, weil sie darauf aufmerksam macht, dass die populäre Vorstellung eines „einsamen Nutzers“ bzw. einer „einsamen Nutzerin“" so nicht stimmt [29, 31, 32]. Zunächst ist die Vorstellung zurückzuweisen, dass das Internet die Bedeutung der Peersozialisation überflügelt, weil eben unter den aktuellen Sozialisationsbedingungen die Peersozialisation von Kindern und Jugendlichen selbst eine immanente digitale Dimension aufweist [3]. Ferner ist die Perspektive des „einsamen Nutzungsverhaltens“ deshalb eingeschränkt, weil die digitale Kommunikation unter Kindern und Jugendlichen oft genug nicht als Ersatz für die Face-to-Face-Kommunikation dient, sondern als ihr selbstverständlicher Bestandteil [33]. Ferner bleibt die individuelle Techniknutzung im Kindes- und Jugendalter notwendig eingebettet in übergreifenden familiale Sozialisationsmuster, die selbst abhängig sind von sozioökonomischen Ressourcen und ethnischen Zugehörigkeiten. So ist es wahrscheinlich, dass im Kindesalter die von den Eltern erlaubte digitale Nutzungszeit innerhalb der Peergroup als Autonomiemaß verhandelt wird und die Frage des frühen eigenen Handybesitzes ein solides Distinktionsmaß darstellt, je nachdem, wer in welchem kindlichen oder jugendlichen Alter mit welcher Generation von Smartphones und welchem allgemeinen Zugriff ausgestattet ist. Die kind- 
liche und jugendliche Distinktionswährung ist die selbstbestimmte, elternunabhängige digitale Techniknutzung. Die Welt der Jugendlichen wiederum ist dermaßen durchdrungen vom Smartphone und der vollständigen Erreichbarkeit [9], dass es begründungsbedürftig ist, sich dem als Jugendlicher entziehen und ein analoges oder zumindest eingeschränkt digitales Leben führen zu wollen.

\section{Digitale Gesundheits- kompetenz und das Verhältnis zu sozialer und gesundheitlicher Ungleichheit}

Wie es oftmals der Fall ist, wird mit der Einführung neuer Technologien die Hoffnung auf Verbesserung aktueller sozialer Missstände verbunden oder darauf hingewiesen, dass sich durch die neuesten technischen Entwicklungen die Gräben sozialer Ungleichheiten eher noch vertiefen. In einer umfassenden Literaturrecherche haben wir gefunden, dass sich der Fokus der Frage nach dem Verhältnis sozialer und digitaler Ungleichheiten im Kontext von Gesundheit in den letzten 15 Jahren, zumindest in hochindustrialisierten Ländern, verschoben hat. Bis in die Mitte der 2000er-Jahre wurde noch die Frage nach dem Zugang in den Mittelpunkt digitaler Ungleichheit, gesundheitsbezogen oder nicht gestellt. Die soziale Ungleichheit wurde als ungleicher Zugang zu den Optionsräumen des Internets operationalisiert [32].

Dieser Fokus hat sich mittlerweile deutlich verschoben. Für die Situation der jugendlichen Lebenswelten in Deutschland wurde unter Verweis auf die JIM-Studie bereits ein hohes Ausmaß an digitaler Durchdringung (Verfügbarkeit und Nutzung digitaler Endgeräte) nachgewiesen. Im Kontext digitaler Ungleichheiten (auch als "Wissenskluft" bzw. „Digital Divide“ bezeichnet [34]) wird deshalb seit Mitte der 2000er-Jahre neben der Frage nach dem Zugang zu Wissen und Information stärker auf die Frage des konkreten Nutzungsverhaltens fokussiert. Ungleichheitsgenerierend ist infolge einer stärkeren Durchdringung nicht nur die mangelnde Verfügbarkeit (First Level Digital Divide), sondern verstärkt die für die Nutzung notwendi- gen Kompetenzen (Second Level Digital Divide) selbst.

In dieser Richtung kommen ganz unterschiedliche Dimensionen sozialer und in der Konsequenz gesundheitlicher Ungleichheit zur Geltung. Zunächst geht es trotz eines prinzipiell vorhandenen digitalen Zugangs darum, ob Kinder und Jugendliche auch in der Lage sind, den Sinn der Informationen, die sie auf Internetseiten finden, zu begreifen. Kim und Xie [35] zeigen in einer systematischen Literaturarbeit, dass mangelhafte Lesbarkeit und Benutzerfreundlichkeit wesentliche Barrieren für den Zugang und Gebrauch von online angebotenen Gesundheitsinformationen darstellen. Hierbei zeigt sich die Relevanz des formalen Bildungsniveaus (Traditional Literacy) von Kindern und Jugendlichen und damit etwa in Deutschland auch die Frage der schulformspezifischen Effekte auf die digitale Gesundheitskompetenz. Gleichzeitig müssen Internetangebote auch den Bedürfnissen der Zielgruppen angepasst werden, sonst, so zeigt das Präventionsdilemma, bleibt die Inanspruchnahme der Maßnahmen aus, was weitere Ungleichheitseffekte zur Folge haben kann [36]. Darüber hinaus müssen die Angebote nicht nur sozialstrukturelle Passung zu den Zielgruppen haben, sondern auch auf deren Bedeutungs- und Sinnzuschreibungsprozesse reagieren, sodass die dargebotenen Informationen realweltliche Bedeutungen für die Adressatinnen und Adressaten haben $[37,38]$.

Ungleichheiten können sich paradoxerweise auch aus einem Zuviel der Nutzung digitaler Angebote ergeben. Hier geht es insbesondere um die Zeit, die Kinder und Jugendliche mit dem Spielen digitaler Spiele, mittlerweile in der Regel online und weltweit, verbringen. Das kann auf speziellen Spielekonsolen, die mittlerweile längst mit dem Internet verbunden sind, erfolgen, an einem normalen PC oder Laptop oder auch an einem mittlerweile handelsüblichen internetfähigen Fernseher. Es liegt eine Reihe von Studien vor, welche darauf hinweisen, dass Kinder und Jugendliche mit niedrigem sozialen Status im Durchschnitt deutlich mehr Zeit vor Bildschirmen (Screen Time) verbringen als sol- che aus Mittel- und Oberschicht (u.a. $[39,40])$. Je länger dabei die Bildschirmzeit ausfällt, desto höher ist das Ausmaß körperlicher Inaktivität [41]. Eine besonders intensive Nutzung der digitalen Welt steht ebenfalls im Widerspruch zur anvisierten digitalen Gesundheitskompetenz. So weisen die Ergebnisse epidemiologischer Studien im Kindes- und Jugendalter auf eine Prävalenz gestörten Medienverhaltens von $10-12 \%$, wobei $1,5-3 \%$ ein abhängiges Verhalten aufweisen [42]. Allerdings muss vor der vorschnellen Anwendung des Klischees eines übermäßigen Konsums digitaler Medien bei Kindern und Jugendlichen der unteren sozialen Klassen gewarnt werden, da es mit einer Reihe von impliziten normativen Vorstellungen vom ,guten (gesundheitsorientierten) Leben" verbunden ist, die höchst problematisch sind [43-45]. Auch die verstärkten Bemühungen, Videospiele zur niedrigschwelligen Förderung von Gesundheit einzusetzen, sollten Beachtung finden [46].

Schließlich ist die klassische verteilungsbezogene Ungleichheitsdimension zu nennen, in der es um die sozial ungleiche Verteilung digitaler Gesundheitskompetenzen geht. Hierzu wurde bisher wenig geforscht. Eine der wenigen aufwendigen Studien stammt aus Israel und untersuchte die Media Health Literacy von 11-, 13- und 15-jährigen Kindern und Jugendlichen. Media Health Literacy ist ein Konstrukt, das aus dem Verständnis von Health Literacy nach Nutbeam [47] und dem Konzept Media Literacy zusammengesetzt ist. Im Rahmen der Studie wurde getestet, wie Media Health Literacy mit Dimensionen des Gesundheitsverhaltens korreliert [3]. Dabei ließ sich feststellen, dass Media Health Literacy bei Mädchen stärker ausgeprägt ist als bei Jungen und dass Kinder und Jugendliche mit einer gebildeten Mutter (mehr als 15 Jahre formale Bildung) über mehr Media Health Literacy verfügen. Allerdings ergaben sich, anders als in vielen Studien zur Gesundheitskompetenz im Erwachsenenalter, keine $\mathrm{Zu}$ sammenhänge zwischen Media Health Literacy und der ethnischen Zugehörigkeit, dem Immigrationsstatus, dem sozioökonomischen Status oder selbst dem Gesundheitsstatus. 
Tab. 1 Instrumente zur Erfassung der digitalen Gesundheitskompetenz (erweitert nach Karnoe und Kayser [51])

\begin{tabular}{|c|c|c|c|c|}
\hline $\begin{array}{l}\text { Titel/ } \\
\text { Referenz }\end{array}$ & $\begin{array}{l}\text { Hintergrund/ } \\
\text { Anwendungsbereich }\end{array}$ & Dimensionen & $\begin{array}{l}\text { Zusammen- } \\
\text { setzung }\end{array}$ & Psychometrische Güte \\
\hline $\begin{array}{l}\text { eHEALS } \\
{[52]}\end{array}$ & $\begin{array}{l}\text { Allgemeine digitale Ge- } \\
\text { sundheitskompetenz, } \\
\text { Selbstbericht, altersgrup- } \\
\text { penübergreifend ein- } \\
\text { setzbar, Adaptationen in } \\
\text { unterschiedliche Sprachen } \\
\text { (u.a. Deutsch) liegen vor }\end{array}$ & $\begin{array}{l}\text { Basiert auf dem Lilienmodell und wird einfaktoriell } \\
\text { konzipiert, in der deutschen Übersetzung lassen sich } \\
2 \text { Faktoren identifizieren: (1) Informationssuche, (2) In- } \\
\text { formationsbewertung }\end{array}$ & $\begin{array}{l}8 \text { Items, 5-stufiges } \\
\text { Antwortformat } \\
\text { (stimme über- } \\
\text { haupt nicht zu bis } \\
\text { stimme völlig zu) }\end{array}$ & $\begin{array}{l}\text { C-Alpha: } 0,88 \text { (Original), } \\
0,83 \text { bis } 0,88 \text { (deutsche } \\
\text { Adaptation), Korrelationen } \\
\text { in moderater Höhe mit } \\
\text { vergleichbaren Konstruk- } \\
\text { ten der Informationssuche } \\
\text { und -bewertung (deutsche } \\
\text { Übersetzung) }\end{array}$ \\
\hline eHLS [54] & $\begin{array}{l}\text { Analyse der mediieren- } \\
\text { den Rolle von digitaler } \\
\text { Gesundheitskompetenz } \\
\text { im Zusammenhang von } \\
\text { individuellen Faktoren } \\
\text { auf das Gesundheitsver- } \\
\text { halten bei Studierenden, } \\
\text { Selbstbericht }\end{array}$ & $\begin{array}{l}\text { Basiert auf dem Verständnis der Fähigkeit des Su- } \\
\text { chens, Findens, Verstehens und Anwendens von ge- } \\
\text { sundheitsbezogenem Wissen. Operationalisierung } \\
\text { über } 3 \text { Dimensionen: (1) funktionale dGK, (2) interakti- } \\
\text { ve dGK, (3) kritische dGK }\end{array}$ & $\begin{array}{l}12 \text { Items, 5-stufi- } \\
\text { ges Antwortformat } \\
\text { (stimme über- } \\
\text { haupt nicht zu bis } \\
\text { stimme völlig zu) }\end{array}$ & $\begin{array}{l}\text { Konfirmatorische Faktor- } \\
\text { analysen bestätigen die } \\
\text { dreifaktorielle Struktur, } \\
\text { keine Angabe zu C-Alpha }\end{array}$ \\
\hline eHLQ [55] & $\begin{array}{l}\text { Basiert auf dem } 7 \text { Di- } \\
\text { mensionen umfassenden } \\
\text { E-Health Literacy Frame- } \\
\text { work, Selbstbericht, Einsatz } \\
\text { vorgesehen für den Be- } \\
\text { reich der Gesundheitsver- } \\
\text { sorgung und kommunale } \\
\text { Settings }\end{array}$ & $\begin{array}{l}\text { Operationalisierung über } 7 \text { Dimensionen: (1) Nutzung } \\
\text { von Technologie zur Verarbeitung von Gesundheitsin- } \\
\text { formationen, (2) Verständnis für Gesundheitskonzepte } \\
\text { und Sprache, (3) Fähigkeit, sich aktiv mit digitalen An- } \\
\text { geboten auseinanderzusetzen, (4) Gefühl der Sicher- } \\
\text { heit und Kontrolle, (5) Motivation zur Auseinanderset- } \\
\text { zung mit digitalen Angeboten, (6) Zugang zu digitalen } \\
\text { Angeboten, (7) Erfüllung individueller Bedürfnisse }\end{array}$ & $\begin{array}{l}35 \text { Items, keine } \\
\text { Angaben zum } \\
\text { Antwortformat }\end{array}$ & $\begin{array}{l}\text { Konfirmatorische Faktor- } \\
\text { analysen bestätigen die } \\
\text { siebenfaktorielle Struktur, } \\
\text { C-Alpha: } 0,77 \text { bis } 0,86, \text { IRT } \\
\text { von } 0,69 \text { bis } 0,96 \text { weisen } \\
\text { auf akzeptable diskrimi- } \\
\text { nante Validität }\end{array}$ \\
\hline DHLI [56] & $\begin{array}{l}\text { Allgemeine digitale Ge- } \\
\text { sundheitskompetenz, } \\
\text { Selbstbericht, altersgrup- } \\
\text { penübergreifend einsetz- } \\
\text { bar }\end{array}$ & $\begin{array}{l}\text { Fokus liegt nicht nur auf der Informationskomponente } \\
\text { (Health 1.0), sondern vor allem auf Interaktion, d.h. } \\
\text { Fähigkeit zur Nutzung und Anwendung (Health 2.0), } \\
\text { Operationalisierung über } 7 \text { Dimensionen: (1) ope- } \\
\text { rative Fähigkeit, (2) Fähigkeit zu Navigation, (3) In- } \\
\text { formationssuche, (4) Bewertung der Verlässlichkeit, } \\
\text { (5) Bestimmung der Relevanz, (6) Inhalt beitragen, } \\
\text { (7) Datenschutz }\end{array}$ & $\begin{array}{l}21 \text { Items, 4-stufi- } \\
\text { ges Antwortformat } \\
\text { (sehr einfach bis } \\
\text { zu sehr schwierig } \\
\text { bzw. nie bis oft) }\end{array}$ & $\begin{array}{l}\text { Identifikation von } 7 \text { Fak- } \\
\text { toren über explorative } \\
\text { Faktoranalyse (aufgeklär- } \\
\text { ter Varianzanteil: } 76 \% \text { ), } \\
\text { C-Alpha: } 0,68 \text { bis } 0,91 \text {, } \\
\text { moderate Korrelation mit } \\
\text { weiteren GK-Instrumenten }\end{array}$ \\
\hline $\begin{array}{l}\text { PRE-HIT } \\
{[57]}\end{array}$ & $\begin{array}{l}\text { Fokus auf Bereitschaft } \\
\text { von Patient*innen mit } \\
\text { chronischen Erkrankun- } \\
\text { gen zum Umgang mit } \\
\text { gesundheitsbezogenen } \\
\text { Informationstechnologien }\end{array}$ & $\begin{array}{l}\text { Operationalisierung über } 8 \text { Dimensionen: (1) Be- } \\
\text { darf an Gesundheitsinformationen, (2) Computer-/ } \\
\text { Interneterfahrung, (3) Computerangst, (4) bevorzug- } \\
\text { ter Interaktionsmodus (5) Fähigkeiten zum Umgang } \\
\text { mit dem Smartphone, (6) Datenschutz im Internet, } \\
\text { (7) keine Nachrichten sind gute Nachrichten }\end{array}$ & $\begin{array}{l}28 \text { Items, 4-stufi- } \\
\text { ges Antwortformat } \\
\text { (stimme über- } \\
\text { haupt nicht zu bis } \\
\text { stimme voll zu) }\end{array}$ & $\begin{array}{l}\text { C-Alpha: } 0,65 \text { bis } 0,87 \text {, } \\
\text { Test/Retest-Reliabilität } \\
\text { ( } 3 \text { Monate): } 0,60 \text { bis } 0,85\end{array}$ \\
\hline \multicolumn{5}{|c|}{$\begin{array}{l}\text { eHEALS eHealth Literacy Scale; PRE-HIT Patient Readiness to Engage in Health Internet Technology; eHLQ eHealth Literacy Questionnaire; eHLS eHealth } \\
\text { Literacy Scale; DHLI Diqital Health Literacy Instrument, } d G K \text { digitale Gesundheitskompetenz, IRT Item Response Theory, GK Gesundheitskompetenz }\end{array}$} \\
\hline
\end{tabular}

\section{Die Messung von digitaler Gesundheitskompetenz}

Ungeachtet ihrer Bedeutung liegen zur Messung von digitaler Gesundheitskompetenz aktuell nur wenige Instrumente vor. Zwar konnten Nguyen et al. [48] in ihrer Übersichtsarbeit 109 Instrumente zur Erfassung von Gesundheitskompetenz ermitteln, jedoch beziehen sich diese nahezu ausschließlich auf den nichtdigitalen Kontext. Für die Altersgruppe der Kinder und Jugendlichen liegen zwei Übersichtsarbeiten vor, welche insgesamt deutlich weniger Verfahren $(n=15-16)$ und dabei keine spezifischen
Instrumente zur digitalen Gesundheitskompetenz identifizieren konnten [49, 50]. Im einzigen uns bekannten Systematisierungsversuch ließen sich acht Instrumente identifizieren, von denen drei Instrumente ausschließlich auf digitale Gesundheitskompetenz ausgerichtet sind und fünf Instrumente Mischkonstrukte aus individueller und digitaler Gesundheitskompetenz darstellen [51]. Davon stellt das „eHEALS“ (E-Health Literacy Scale) das im internationalen Kontext am häufigsten eingesetzte Instrument dar (•Tab. 1). Hierbei handelt es sich um eine Operationalisierung des Lilienmodells durch Norman und
Skinner [52] mittels acht Items, die auf einer fünfstufigen Antwortskala beantwortet werden können. Mittlerweile liegen zahlreiche Übersetzungen und Validierungsstudien vor, wobei sich in der deutschsprachigen Adaptation entgegen der ursprünglich vorgeschlagenen Eindimensionalität zwei Faktoren (Informationssuche, Informationsbewertung) ableiten lassen [53]. Die Häufigkeit des Einsatzes des eHEALS-Fragebogens steht aus unserer Sicht jedoch kaum in einem sinnvollen Verhältnis zu der mit dem Konzept digitaler Gesundheitskompetenz angeschnittenen Komplexität. Dennoch wird der eHEALS-Fragebogen bis 
heute als adäquates Instrument zur Messung digitaler Gesundheitskompetenzen eingeschätzt [35].

Ohne Anspruch auf Vollständigkeit finden sich in 0 Tab. 1 noch vier weitere Instrumente zur Erfassung der digitalen Gesundheitskompetenz. Während mit dem PRE-HIT (Patient Readiness to Engage in Health Internet Technology [57]) und dem eHLQ (eHealth Literacy Questionnaire [55]) Verfahren vorliegen, die einen stärkeren Fokus auf Zielgruppen der Gesundheitsversorgung (z.B. Patientinnen und Patienten) aufweisen, zeichnen sich das eHLS (eHealth Literacy Scale [54]) und das DHLI (Digital Health Literacy Instrument [56]) durch ihre zielgruppenübergreifende Ausrichtung aus. Vor allem das jüngst von van der Vaart und Drossaert [56] vorgelegte DHLI ist gegenüber dem eHEALS mit insgesamt 21 Items deutlich umfangreicher. Dabei stehen, neben der „klassischen“ Informationskomponente, Fragen der Interaktion, d.h. der Fähigkeit zur Nutzung und Anwendung, im Vordergrund. Faktoranalytisch ließen sich dabei 7 Dimensionen identifizieren: (1) operative Fähigkeit, (2) Fähigkeit zu Navigation, (3) Informationssuche, (4) Bewertung der Verlässlichkeit, (5) Bestimmung der Relevanz, (6) Inhalt beitragen und (7) Datenschutz. Die Erprobung im deutschsprachigen Raum und für Jugendliche steht bislang noch aus, ist jedoch zumindest teilweise in einem laufenden Projekt der Hochschule Fulda (DiGKS, digitale Gesundheitskompetenz von Schülerinnen und Schülern) vorgesehen.

Obgleich mittlerweile unterschiedliche Screeninginstrumente vorliegen, wird die digitale Gesundheitskompetenz bisher nicht umfassend genug, also in all ihren Einzelkompetenzen abgebildet [35]. Nach unserem Kenntnisstand existiert insbesondere kein Instrument, das die digitalen Gesundheitskompetenzen als soziale Praxis und deren mögliche Verfügbarkeit im sozialen Raum („distributed health literacy“ $[58,59])$ hinreichend erfasst, weder bei Erwachsenen noch bei Kindern und Jugendlichen. Das ist insofern problematisch, da, wie bereits angedeutet, die Vorstellung einsamer Techniknutzung mit den kindlichen und jugendlichen Lebenswelten nur sehr bedingt etwas zu tun hat. Dieser Mangel verweist auf ein systematischeres Problem innerhalb der konzeptionellen und empirischen Health-Literacyund E-Health-Literacy-Forschung, die mit handlungstheoretischer Grundlegung in aller Regel subjektorientierter Gesundheitsentscheidungen und der implizit verwendeten kognitivistischen Grundierung zu tun hat. In zahlreichen Veröffentlichungen wird darauf hingewiesen, dass für die Messung von Health Literacy bestimmte Kriterien definiert werden sollten, die als theoretische Hilfestellungen für die Entwicklung von Fragebögen herangezogen werden sollten. Hierzu zählen beispielsweise ein zuvor festgelegtes Modell, eine eindeutig zugrunde liegende Definition und realistisch messbare Teilkomponenten [60-62]. Auch für Messinstrumente im Kindes- und Jugendalter liegen solche Hinweise bereits vor $[46,63]$.

\section{Schlussbetrachtung: von der Deskription zur Intervention}

Vor dem Hintergrund einer hohen Alltagsdurchdringung und einer täglichen Nutzung von durchschnittlich mehr als drei Stunden [64] spielen digitale Medien auch für gesundheitsbezogene Belange eine zunehmend bedeutsame Rolle. Im Rahmen des Beitrags wurde argumentiert, dass infolge der guten $\mathrm{Zu}$ gänglichkeit und der Allgegenwärtigkeit von Gesundheitsinformationen ein hohes Ausmaß an individuellen Fähigkeiten gefordert ist, geeignete Informationen auszuwählen und deren Verlässlichkeit einzuschätzen. Hinzu kommt, dass die Mediennutzenden nicht nur passiv rezipieren, sondern auch aktiv an der Generierung und Verbreitung gesundheitsbezogener Informationsinhalte teilhaben sollten. Digitale Gesundheitskompetenz bedeutet in diesem Sinne nicht nur, Botschaften aufzunehmen und zu verarbeiten, sondern schließt die Fähigkeit mit ein, angemessen am multidirektionalen digitalen Kommunikationsgeschehen teilzunehmen. Entsprechende Interaktionskompetenzen sind bislang vergleichsweise selten in der konzeptionell-theoretischen Diskussion vertreten (z. B. $[56,58,59])$. Eine weitere konzeptionelle Unklarheit besteht im Verhältnis zwischen Gesundheitskompetenz und digitaler Gesundheitskompetenz. Wenngleich Gesundheitskompetenz im Lilienmodell eine zentrale Dimension von digitaler Gesundheitskompetenz darstellt, ist die empirische Studienlage zum Zusammenhang beider Konstrukte (in Abhängigkeit von den Instrumenten und Stichproben) bislang heterogen [65, 66].

Ohnehin mangelt es derzeit noch an einer ausreichenden empirischen Basis, um die Ausprägung der digitalen Gesundheitskompetenz insbesondere von Heranwachsenden in Deutschland angemessen abschätzen zu können. Dieser Mangel gilt gleichermaßen für die theoretisch-konzeptionelle Basis, denn der Health-Literacy-Diskurs ist bislang fern davon, ein einheitliches Modell präsentieren zu können. Dies mag sicherlich ein Grund für den aktuellen Mangel an deutschsprachigen Interventionen zur Förderung der digitalen Gesundheitskompetenz sein. Anderseits ist infolge der beschriebenen konzeptionellen Verschränkung zu berücksichtigen, dass im Bereich der Medienkompetenzförderung bereits zahlreiche Aktivitäten unternommen werden. Exemplarisch angeführt werden kann das positiv evaluierte Programm „Medienhelden“, welches auf die Prävention von Cybermobbing und die Förderung von Internet- und Medienkompetenz bei Schülerinnen und Schülern der Klassenstufe 7 bis 10 abzielt [67]. Es bedarf in Zukunft sicherlich mehr Maßnahmen, in denen Medienund Gesundheitskompetenzen systematisch miteinander verknüpft werden. Hierbei stellt die Schule ein geeignetes Umsetzungssetting dar, dies nicht nur, weil Kinder und Jugendliche aufgrund der Schulpflicht dort einen hohen Anteil ihres Tages verbringen, sondern vor allem, weil mit der Strategie „Bildung in der digitalen Welt" ein Kompetenzrahmen mit 6 übergeordneten Kompetenzbereichen und 22 dazugehörigen Kategorien durch die Kultusministerkonferenz verabschiedet wurde [18]. Dieser stellt eine wichtige Referenz dar, an der sich auch Maßnahmen zur Stärkung von digitaler Gesundheitskompetenz ausrichten 
sollten, um nachhaltig Eingang in das Setting Schule zu finden. Dabei sollte im Sinne der ganzheitlichen Ausrichtung des Konzepts die strukturelle Dimension nicht unberücksichtigt bleiben. Gerade im schulischen Kontext gehört neben der Verbesserung der infrastrukturellen Rahmenbedingungen (u.a. durch den DigitalPakt Schule von Bund und Ländern [68]) ebenfalls die Förderung von Medienkompetenzen bei Lehrkräften und weiteren Fachkräften dazu. Auch hierfür liegt mit dem European Framework for the Digital Competence of Educators ein erster Rahmen vor [69].

\section{Korrespondenzadresse}

\section{Prof. Dr. Uwe H. Bittlingmayer}

Institut für Soziologie, Pädagogische

Hochschule Freiburg

Kunzenweg 21, 79117 Freiburg, Deutschland

uwe.bittlingmayer@ph-freiburg.de

Funding. Open Access funding provided by Projekt DEAL.

\section{Einhaltung ethischer Richtlinien}

Interessenkonflikt. U.H. Bittlingmayer, K. Dadaczynski, D. Sahrai, S. van den Broucke und O. Okan geben an, dass kein Interessenkonflikt besteht.

Für diesen Beitrag wurden von den Autoren keine Studien an Menschen oder Tieren durchgeführt. Für die aufgeführten Studien gelten die jeweils dort angegebenen ethischen Richtlinien.

Open Access. Dieser Artikel wird unter der Creative Commons Namensnennung 4.0 International Lizenz veröffentlicht, welche die Nutzung, Vervielfältigung, Bearbeitung, Verbreitung und Wiedergabe in jeglichem Medium und Format erlaubt, sofern Sie den/die ursprünglichen Autor(en) und die Quelle ordnungsgemäß nennen, einen Link zur Creative Commons Lizenz beifügen und angeben, ob Änderungen vorgenommen wurden.

Die in diesem Artikel enthaltenen Bilder und sonstiges Drittmaterial unterliegen ebenfalls der genannten Creative Commons Lizenz, sofern sich aus der Abbildungslegende nichts anderes ergibt. Sofern das betreffende Material nicht unter der genannten Creative Commons Lizenz steht und die betreffende Handlung nicht nach gesetzlichen Vorschriften erlaubt ist, ist für die oben aufgeführten Weiterverwendungen des $\mathrm{Ma}$ terials die Einwilligung des jeweiligen Rechteinhabers einzuholen.

Weitere Details zur Lizenz entnehmen Sie bitte der Lizenzinformation auf http://creativecommons.org/ licenses/by/4.0/deed.de.

\section{Literatur}

1. Del Giudice P (2017) The IC-health project: improving digital health literacy in Europe. Eur J Public Health 27(3):26

2. Norman CD, Skinner HA (2006) ehealth literacy: essential skills for consumer health in a networked world. J Med Internet Res 8(2):e9

3. Levin-Zamir D, Lemish D, Gofin R (2011) Media health literacy (MHL): development and measurement of the concept among adolescents. Health Educ Res 26(2):323-335

4. Seçkin G, Yeatts D, Hughes S, Hudson C, Bell V (2016) Being an informed consumer of health information and assessment of electronic health literacy in a national sample of Internet users: validity and reliability of the e-HLS instrument. JMed Internet Res 18(7):e161

5. Robinson C, Graham J (2010) Perceived Internet health literacy of HIV-positive people through the provision of a computer and Internet health education intervention. Health Info Libr J 27(4):295-303

6. Vaz NFM (2018) Mobile health literacy to improve health outcomes in low-middle income countries. In health care delivery and clinical science: concepts, methodologies, tools, and applications, S1398-1411

7. Sørensen K, Van den BrouckeS, Fullam Jet al (2012) Health literacy and public health: a systematic review and integration of definitions and models. BMC Public Health 12:80

8. Begoray DL, Banister EM, Wharf Higgins J, Wilmot R (2014) Online, tuned in, turned on: multimedia approaches to fostering critical media health literacy for adolescents. Asia-Pacific J Health Sport Phys Educ 5(3):267-280

9. Inchley J, Currie D, Young T et al (Hrsg) (2016) Growing up unequal: gender and socioeconomic differences in young people's and well-being. Health behaviour in school-aged children (HBSC) study: international report from the 2013/2014 survey. Health policy for children and adolescents, no. 7. World Health Organization, Regional Office for Europe, Copenhagen

10. Intarakamhang U, Intarakamhang P (2017) Health literacy scale and causal model of childhood overweight. JRes Health Sci 17(1):e368

11. Manganello J (2008) Health literacy and adolescents: a framework and agenda for future research. Health Educ Res 23(5):840-847

12. Okan O, Rowlands G, Sykes S, Wills J (2020) Shaping alcohol health literacy: a systematic concept analysis and review. Health Lit Res Pract 4(1):e3-e20. https://doi.org/10.3928/2474830720191104-01

13. Wharf Higgins J, Begoray D (2012) Exploring the borderlands between media and health: conceptualizing "critical media health literacy". JMedia LitEduc 4(2):136-148

14. WHO-World Health Organization (2015) Health 2020: education and health through the lifecourse. World Health Organization, Regional Office for Europe, Copenhagen

15. Zarcadoolas C, Pleasant A, Greer DS (2005) Understanding health literacy: an expanded model. Health Promot Int 20(2):195-203

16. UNICEF—United Nations Children's Fund (2017) The state of the world's children 2017-children in a digital world. https://www.uniceforg/ publications/index_101992.html.Zugegriffen: 14. Juli2019
17. KMK - Kultusministerkonferenz (2012a) Medienbildung in der Schule. Beschluss der Kultusministerkonferenzvom 08.03.2012. KMK, Berlin

18. KMK - Kultusministerkonferenz (2016in) Bildung in der digitalen Welt:Strategie der Kultusministerkonferenz. Beschluss der Kultusministerkonferenz vom 08.12.2016 in der Fassung vom 07.12.2017. KMK, Berlin

19. Feierabend S, Rathgeb T, Reutter T (2018) JIM Studie 2018. Jugend, Information, Medien. Basisuntersuchung zum Medienumgang 12- bis 19Jähriger. Medienpädagogischer Forschungsverbund Südwest, Stuttgart

20. Feierabend S, Rathgeb T, Reutter T (2018) KIM Studie 2018. Kindheit, Internet, Medien. Basisuntersuchung zum Medienumgang 6- bis 13Jähriger. Medienpädagogischer Forschungsverbund Südwest, Stuttgart

21. Park E, Kwon M (2018) Health-related Internet use by children and adolescents: systematic review. JMed Internet Res 20(4):e120

22. Syed-Abdul S, Fernandez-Luque L, Jian WS et al (2013) Misleading health-related information promoted through video-based social media: anorexia on youtube. J Med Internet Res 15(2):e30

23. TNSPolitical\&Social, European Commission (2014) Flash Eurobarometer 404: European citizens' digital health literacy. http://ec.europa.eu/public opinion/flash/fl_404_en.pdf. Zugegriffen: 19. Aug. 2019

24. Paakkari LT, George S (2018) Ethical underpinnings for the development of health literacy in schools: ethical premises ('why'), orientations ('what'). BMC Public Health 18(1):326

25. Bröder J, Okan O, Bauer U, Bruland D et al (2017) Health literacy in childhood and youth: a systematic review of definitions and models. BMC Public Health 17:361

26. Borzekowski DLG (2009) Considering children and health literacy: a theoretical approach. Pediatrics 124:S282-S288

27. Eng TR (2001) The e-health landscape: a terrain map of emerging information and communication technologies in healthand health care. The Robert Wood Johnson Foundation, Princeton, NJ

28. Baacke D (1997) Medienkompetenz. Niemeyer, Tübingen

29. Bautista JR (2015) From solving a health problem to achieving quality of life: redifining eHealth literacy. Jit Technol 16(2):33-54

30. Gilstad H (2014) Toward a comprehensive model of ehealth literacy. In: Jaatun EAA, Brooks E, Berntsen KE, Gilstad H, Jaatun MG (Hrsg) Proceedings of the 2nd European workshop on practical aspects of health Informatics

31. Michikyan M, Suárez-Orozco C (2016) Adolescent media and social media use: implications for development.J Adolesc Res 31(4):411-414

32. Bittlingmayer UH, Islertas Z, Sahrai E et al (2020) Health Literacy aus gesundheitsethnologischer Perspektive. Eine Analyse alltäglicher Gesundheitspraktischen von migrantischen Jugendlichen und Familien. SpringerVS, Wiesbaden

33. Suter L, Waller G, Bernath J, Külling C, Willemse I, Süß D (2018) JAMES - Jugend, Aktivitäten, Medien - Erhebung Schweiz. Zürcher Hochschule für Angewandte Wissenschaften, Zürich

34. Bonfadelli H (2018) Soziale Ungleichheit: Nutzung und Wirkung von Gesundheitsinformationen im Internet. In: Scherenberg V, Pundt J (Hrsg) Digitale Gesundheitskommunikation zwischen Meinungsbildung und Manipulation. Apollon University Press, Bremen, S87-99 


\section{Leitthema}

35. Kim H, Xie B (2017) Health literacy in the ehealth era: a systematic review of the literature. Patient Educ Couns 100(6):1073-1082

36. Bauer U (2005) Das Präventionsdilemma: Potenziale schulischer Kompetenzförderung im Spiegel sozialer Polarisierung. VS, Wiesbaden

37. Fairbrother H, Curtis P, Goyder E (2016a) Making health information meaningful: children's health literacy practices. SSM Popul Health 2:476-484

38. Pinheiro $P(2019)$ Future avenues for health literacy: learning from literacy and literacy learning. In: Okan O, Bauer U, Levin-Zamir D, Pinheiro $\mathrm{P}$ Sørensen K (Hrsg) International handbook of health literacy. Research, practice and policy across the lifespan. Policy Press, Bristol, S555-572

39. Fairclough SJ, Boddy LM, Hackett AF, Stratton G (2009) Associations between children's socioeconomic status, weight status, and sex, with screen-based sedentary behaviours and sport participation. Int JPediatr Obes 4(4):299-305

40. Robert Koch-Institut (Hrsg) (2015) Mediennutzung. Faktenblatt zu KiGGS Welle 1: Studie zur Gesundheit von Kindern und Jugendlichen in Deutschland - Erste Folgebefragung 2009-2012. RKI, Berlin

41. Manz K, Schlack R, Poethko-Müller C et al (2014) Körperlich-sportliche Aktivität und Nutzung elektronischer Medien im Kindes-und Jugendalter. Bundesgesundheitsblatt Gesundheitsforschung Gesundheitsschutz 57(7):840-848

42. Bilke-Hentsch O (2017) Pathologische Mediennutzung bei Kindern und Jugendlichen - eine Perspektive für die Praxis. Praxis 106(14):775-779

43. Bittlingmayer UH, Ziegler H (2012) Public Health und das gute Leben. Der Capabilities-Approach als normatives Fundament interventionsbezogener Gesundheitswissenschaften? Wissenschaftszentrum Berlin für Sozialforschung (WZB), Berlin (WZB Discussion Paper, No. SPI2012-301)

44. Schmidt B (2017) Exklusive Gesundheit. Gesundheit als Instrument zur Sicherstellung sozialer Ordnung. SpringerVS, Wiesbaden

45. Kühn H (1993) Healthismus. Eine Analyse der Präventionspolitik und Gesundheitsförderung in den USA. edition sigma, Berlin

46. Dadaczynski K, Schiemann S, Paulus P (Hrsg) (2016) Gesundheit spielend fördern: Potenziale und Herausforderungen von digitalen Spieleanwendungen für die Gesundheitsförderung und Prävention. Beltz Juventa, Weinheim

47. Nutbeam D (2000) Health literacy as a public health goal: a challenge for contemporary health education and communication strategies into the 21st century. Health Promot Int 15(3):259-267

48. Nguyen TH, Park H, Han HR et al (2015) State of the science of health literacy measures: validity implications forminority populations. PatientEduc Couns 98(12):1492-1512

49. Okan O, Lopes E, Bollweg TM et al (2018) Generic health literacy measurement instruments for children and adolescents: a systematic review of the literature. BMC Public Health 18:166

50. Ormshaw MJ, Paakkari LT, Kannas LK (2013) Measuring child and adolescent health literacy: a systematic review of literature. Health Educ 113(5):433-455

51. Karnoe A, Kayser L (2015) How is eHealth literacy measured and what do the measurments tell us? A systematic review. Knowl Manag E Learn 7(4):576-600

52. NormanCD, SkinnerHA(2006)eHEALS: the ehealth literacy scale. J Med Internet Res 8(4):e27
53. Soellner R, Huber S, Reder M (2014) The concept of eHealth literacy and its measurement. J Media Psychol 26:29-38

54. Hsu W, Chiang C, Yang S (2014) The effect of individual factors on health behaviors among college students: the mediating effects of eHealth literacy. J Med Internet Res 16(12):e287

55. Kayser L, Karnoe A, Furstrand D et al (2018) A multidimensional tool based on the ehealth literacy framework: development and initial validity testing of the ehealth literacy questionnaire (eHLQ). J Med Internet Res 20(2):e36

56. van der Vaart R, Drossaert C (2017) Development of the digital health literacy instrument: measuring a broad spectrum of health 1.0 and health 2.0 skills. JMed Internet Res 19(1):e27

57. Koopman RJ, Petroski GF, Canfield SM, Stuppy JA Mehr DR (2014) Development of the PRE-HIT instrument: patient readiness to engage in health information technology. BMC Fam Pract 15(1):18

58. Papen U (2009) Literacy, learning and health-a social practices view of health literacy. Lit Numer Stud. https://doi.org/10.5130/Ins.v0i0.1275

59. Edwards $M$, Wood F, Davies M, Edwards A (2015) 'Distributed health literacy': Iongitudinal qualitative analysis of the roles of health literacy mediators and social networks of people living with a long-term health condition. Health Expect 18(5):1180-1193

60. Pleasant A, McKinney J, Rikard RV (2011) Health literacy measurement: a proposed research agenda. J Health Commun 16(3):11-21

61. Pleasant A (2014) Advancing health literacy measurement: a pathway to better health and health system performance. J Health Commun 19(12):1481-1496

62. Pleasant A, Rudd RE, O'Leary C et al (2016) Considerations for a new definition of health literacy. Discussion paper. National academy of medicine. https://nam.edu/wp-content/uploads/ 2016/04/Considerations-for-a-New-Definitionof-Health-Literacy.pdf. Zugegriffen: 14. Juli 2019

63. Bollweg TM, Okan O (2019) Measuring children's health literacy:current approaches and challenges. In: Okan O, Bauer U, Levin-Zamir D, Pinheiro P, Sørensen K (Hrsg) International handbook of health literacy. Research, practice and policy across the lifespan. Policy Press, Bristol, S83-98

64. Frees B, Koch W (2018) ARD/ZDF-Onlinestudie 2018: Zuwachs bei medialer Internetnutzung und Kommunikation, S398-413

65. Duplaga M, Grysztar M, Tubek A (2017) The association between health literacy and ehealth literacy in young adult population in Poland. Eur J Public Health 27(3):432

66. Monkmana H, Kushniruka AW, Barnetta J, Boryckia EM, Greinerb LE, Sheetsc D (2018) Are health literacy and ehealth literacy the same or different? In: Gundlapalli AV, Jaulent MC, Thao D (Hrsg) MEDINFO 2017: precision healthcare through Informatics: proceedings of the 16th world congress on medical and health Informatics. IOS Press, Amsterdam (Vol. 245, 178-182)

67. Schultze-Krumbholz A, Schultze M, Zagorscak P, Wölfer R, Scheithauer H (2016) Feeling cybervictims' pain-the effect of empathy training on cyberbullying. Aggress Behav 42:147-156

68. Bundesministerium für Bildung und Forschung (2019) DigitalPakt Schule ist gestartet. https:// www.bildung-forschung.digital/de/digitalpaktschule-ist-gestartet-2552.html. Zugegriffen: 19. Aug. 2019
69. Redecker C (2017) European framework for the digital competence of educators. Publications Office of the European Union, Luxembourg 